\title{
Whale Optimization Algorithm Based CME-CV Thresholding for Soil Porous Medium Extraction
}

\author{
Arunpandian Murugan, Arunprasath Thiyagarajan, Pallikonda Rajasekaran Murugan, Vishnuvarthanan \\ Govindaraj, Vigneshwaran Senthilvel
}

\begin{abstract}
Simulated Soil CT image is one of the rich boundary limitation of all the informations of soil pore, pebble and solid medium. Ground truth of soil skeleton is more useful to examine porosity and void ratio. With the help of the above parameters are used to recognize the other physical natures of soil. Soil management system is a well-known identified tool to enhance the field of work to do. Image processing is the major methods to examine and delivers the outcomes. Primary process of image processing is thresholding the given input samples and limits the boundary of the various objects. Thresholding is under the trustworthy of most powerful two traditional algorithms such as Otsu inter class variance and Kapur's maximum entropy. Mixture of both the traditional methods are given all the informations of soil samples. In proposed towards novelty, combine some Bio- Inspired optimization with the traditional mixtures. Whale Optimization Algorithm (WOA) enhance the efficiency and accuracy of the segmentation throughput. It compares between certain conventional Bio-inspired Optimization.
\end{abstract}

Keywords: Image thresholding, WOA, PSO, ABC and CME-CV, Otsu class variance and Kapur maximum entropy.

\section{INTRODUCTION}

In Soil managing system, Soil porosity and void ratio are major impact for selection of work on the field. In image based porosity analysis, Estimation of soil porosity and void ratio has required some more enlargements to prove their efficiency [12]. Metahueristic optimization has more impact on over the last two decades. It gives enormous changes in accordance with the efficient and precision. Void ratio and porosity of the samples are major definer of the morphological skeleton and microbial growth of roots and water capability of soil samples [14]. Roy and Perfect et al., to examine the lacunarity and transport processes with representations of soil combinations using analytical and digital tools [4]. Thresholding is remarkable and more effective part in image segmentation of both the global or single objective and local or multi-objective segmentation [5].

Revised Manuscript Received on December 16, 2019.

Mr. M. Arunpandian, Research Scholar, Department of Electronics and Communication Engineering, Kalasalingam Academy of Research and Education.

Dr. T. Arunprasath, Associate Professor, Department Biomedical Engineering, Kalasalingam Academy of Research and Education.

Dr. M. Pallikonda Rajasekaran, Professor in 2012 in Kalasalingam Academy of Research and Education.

Dr. G. Vishnuvarthanan,Associate Professor with the Department of Biomedical Engineering of School of Bio and Chemical Sciences in the Kalasalingam Academy of Research and Education, Tamilnadu, India.

Vigneshwaran Senthilvel, Department of Biomedical Engineering of School of Bio and Chemical Sciences in the Kalasalingam Academy of Research and Education, Tamilnadu, India.
Otsu et al. proposed an optimal threshold value selection by the maximization of inter-class variance or the minimization of intra class variance between the two objects. Sahoo et al. suggested the alternative solution for finding the cost effective threshold value using the maximization of entropy of an image. In the reason behind the complications of effective threshold value selection, researchers introduced some naturally inspired optimization algorithms for improving the accuracy and efficiency to pick the optimal value. According to the Darwinian law, framed the Genetic Algorithm (GA) to improve the efficiency of a normal thresholding based segmentation. In the same way, some traditional algorithms such as PSO and ABC inspired their functions likewise. In the evolution of swarm based optimization approaches are getting further enormous modifications towards the best value chosen. In which, it is used to produce the finest solution for the complicated problems. The foraging behaviour of the ants was inspiring for the Ant Colony Optimization (ACO). From the matting behaviour of honey bees was the inspiration for Honey Bee Matting Optimization (HBMO) approaches. SSA is newly established standard optimization algorithm. WOA Optimization algorithm is used to produce a high efficient solutions for more complicated problems. Analysis is given in section 3, a comparative analysis of WOA based CME-CV with PSO based CME-CV and ABC based CME-CV. In these comparisons are evaluated by the following parameters such porosity and Void ratio of the simulated soil CT samples. This evaluation elucidate their accuracy using such segmentation parameters relative soil porosity and misclassification errors. The efficacy of the author offered WOA based CME-CV approach is detailed in the forthcoming sections.

\section{MATERIALS AND METHODOLOGY}

In CT soil samples having more information of each and every macro and micro soil pores when compare to the other modality of digital image samples. In general soil CT samples specifies all the information of soil macro pores, micro pores and pebbles spaces. Using truncated multi-fractal analysis, it simulate a 2-D CT soil sample in various user specified characterization of macro, micro and pebble spaces. It categorized into 5 different ranges based on the porosity level of simulated soil samples and named as range-1, 2, 3, 4 and 5.
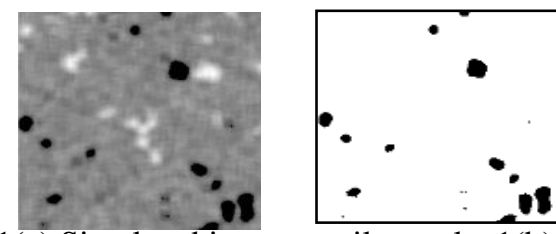

Fig 1: 1(a) Simulated input ct soil sample. 1(b) ground truth 
In fig-1.a and Fig-1.b shows the simulated CT soil image with the porosity level of $0-5.0 \%$ and its skeleton of simulated soil CT samples. It generated simulated soil CT samples are under the following specifications of 24-bit grey scale image with the dimensions of $256 \times 256$ pixels.

Table-1: Mean And Standard Deviation Of The Simulated Soil Samples

\begin{tabular}{|l|l|l|l|}
\hline Pore limit & $\begin{array}{l}\text { No.of Simulated } \\
\text { samples }\end{array}$ & $\begin{array}{l}\text { Mean porosity of } \\
\text { simulated samples } \\
(\%)\end{array}$ & $\begin{array}{l}\text { Standard deviation of } \\
\text { simulated samples } \\
(\%)\end{array}$ \\
\hline Limit-1 (0-5\%) & 10 & 3.31 & 0.96 \\
\hline Limit-2 (5-10\%) & 10 & 7.72 & 1.6 \\
\hline Limit-3(10-15\%) & 10 & 13.16 & 1.15 \\
\hline Limit-4(15-20\%) & 10 & 17.72 & 1.49 \\
\hline Limit-5(20-25\%) & 10 & 22.76 & 1.53 \\
\hline
\end{tabular}

\section{A.Simulated soil sample selection}

Generation procedure for simulated soil sample

From the vast survey, using Truncated Multi-fractal method is to simulate soil CT image with the characterization of data samples used in. It is full of business on grayscale simulated images with the following characteristics.

- It needs to have clear bound of histogram values.

- It reflect the pores and solid pixels with the individual properties. space.

- It must be show its reliability of soil sample image.

\section{B.Procedure for simulated CT soil image structural design}

1. Using Siperenski Multi-fractal method is to allaocte the various sapces present in the soil sample.

2. User specified a certain histogram level for the particular contents in simulated soil samples. Average pore space $=0$. It results to generate ground truth of simulated soil samples.

3. To improve reliability of an image, we added Gaussian white noise. It will reduce the high resolution.

4. To reduce the high contrast image sample by using low pass filter.

\section{C.Thresholding}

Thresholding is a type of raw separation of foreground and background objects in digital image samples. It simply converts the grey scale samples into desired binary samples with rich useful information. It categorizes based on the number of objects present as Bi-model and Multi-model thresholding. Here, the most trusted and traditional Combination of both Maximum Entropy and Class Variance thresholding is elucidated in the following sessions [3].

\section{D.Combined Maximum Entropy and Class Variance thresholding}

Otsu [1], [10] introduces a most reliable optimal thresholding to separates image samples into foreground and background information. It clarifies and bounds object information either maximizes inter-class variance or intra-class variance
- Clearly designed and differentiate the pebble and pore

between neighbouring pixels. Kapur [2] introduces another mode of raw separation of foreground and background pixels. Entropy is a quantity of image information in various pixel intensity level. The optimal threshold value of image is evaluated from either maximizes or minimizes of entropy function. Using the above thresholding methodologies are provides the minimum segmentation efficacy either neighbouring spaces or edges. To overcome the above mentioned problem in traditional methodologies, arunpandian et al. [13] have used combines both the maximum entropy and also maximizes the interclass variance, which offers better segmentation efficacy in neighbouring and also edge pixels. In equation (10), it provides the unique optimal pixel, which maximizes both the entropy and interclass variance. Successfully, CME-CV approach is given effective evaluation of pore space and void ratio of simulated soil samples [13].

Optimal threshold value

$$
\mathrm{F}=\arg \max \left\{w_{0}(k) * \sigma_{0}^{2}(k)+w_{1}(k) * \sigma_{1}^{2}(k)+\right.
$$$$
\left.\mathrm{H}_{\mathrm{a}}+\mathrm{H}_{\mathrm{b}}\right\}
$$

(1)

From equation (1), F represents the unique optimal threshold value and $H_{a}$, and $H_{a}$ are the entropy of two different grey levels values of dissimilar objects respectively. $\sigma_{0}^{2}$ and $\sigma_{1}^{2}$ are the class variances between two different classes. Further consideration of efficiency and accuracy of outcomes, the author have intended with some metaheuristic optimization and decision making techniques. Bio-inspired optimization approach (metaheuristic optimization) is a very close learning of social behaviour and emergence of the particular organisms that otherwise known as biologically inspired optimization. It have numerous categories based on the any behaviour of particular animal or bird or any organisms. Here, this analysis reveals that the effectiveness of the proposed algorithm delivers better outcomes when compared with some metaheuristic optimization algorithms such as Particle Swarm Optimization (PSO), Artificial Bee Colony Optimization (ABC) and Whale Optimization Algorithm (WOA). The author have propounded the Whale Optimization Algorithm that the objective function supplies high convergence and coverage area. 


\section{E. Whale optimization Algorithm}

Miraliji et al., Suggested a novel metaheuristic optimization technique, which is inspired by the foraging behavior of humpback whales. It hunts the group of small fishes in the two different ways such as encircling prey and spiral bubble net attacking methods. Initially, it encircles the prey to hunt using the following equations,

$$
\begin{array}{r}
D=\left|C \cdot \overrightarrow{X^{*}(t)}-X(t)\right| \\
X(t+1)=X^{*}(t)-\vec{A} \cdot D
\end{array}
$$

where $\mathrm{t}$ indicates the current iteration, $\mathrm{X} *$ represents the best solution obtained so far, $X$ is the position vector, || is the absolute value, and · is an element-by-element multiplication. In addition, $\mathrm{A}$ and $\mathrm{C}$ are coefficient vectors that are calculated as in Equations (4) and (5), respectively:

$$
\vec{A}=\overrightarrow{2 a} \cdot \vec{r}-\vec{a}
$$

$$
C=2 . \vec{r}
$$

Where a decreases linearly from 2 to 0 over the course of iterations (in both exploration and exploitation phases) and $r$ is a random vector generated with uniform distribution in the interval of $[0,1]$. According to Eq. (3) the search agents (whales) update their positions according to the position of the best known solution (prey). The adjustment of the values of $\mathrm{A}$ and $\mathrm{C}$ vectors control the areas where a whale can be located in the neighborhood of the prey.

\section{Algorithm of WOA based CME-CV}

Step-1: Feed input sample to the system

Step-2: Calculate the fitness value of each solution.

Step-3: Assign the Maximum number of Iterations

Step-4: update the values of a, A, 1 and C.

Step-5: Randomly search the agents

Step-6: Update the new position using equation (4).

Step-7: Repeat step-3 to Step-6 until meet your requirements.

\section{Results and Discussion:}

In this section, experimental results are analyzed from table 2-3. In table-1 denotes the various optimal threshold value for the four different kinds such as CME-CV, PSO based CME-CV, ABC based CME-CV and WOA based CME-CV. Four different approaches give four different values of optimal threshold and porosity and provides void ratio with respect to the optimal threshold value. All the four approaches of porosity of simulated soil sample are compared with their ground truth, where it is simulated using truncated fractal analysis.

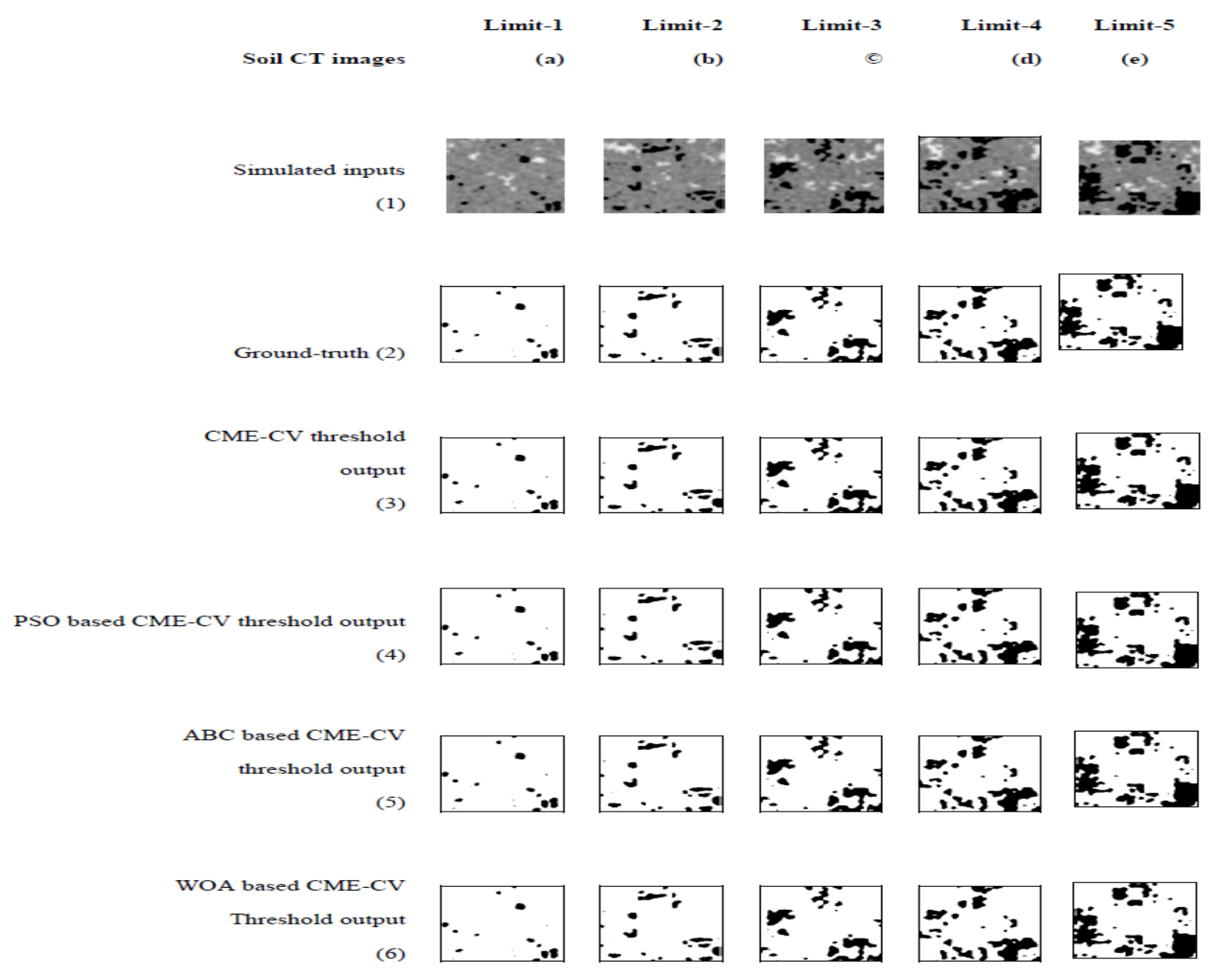

Fig 4: Simulated soil ct input samples. (1). Simulated soil CT samples. (2). Ground-truth of soil CT images. (3). CME-CV threshold based outputs. (4) pso based CME-CV outputs (5). Abc based cme-cv outputs. (6) WOA based CME-CV outputs. 
Whale Optimization Algorithm Based CME-CV Thresholding for Soil Porous Medium Extraction

Table-3: Comparison of porosity and void ratio of simulated soil samples

\begin{tabular}{|c|c|c|c|c|c|c|c|c|c|}
\hline \multirow[b]{2}{*}{ INPUT } & \multirow{2}{*}{$\begin{array}{l}\text { GT-POROSITY } \\
(\%)\end{array}$} & \multicolumn{4}{|c|}{ POROSITY (\%) } & \multicolumn{4}{|c|}{ VOID RATIO (\%) } \\
\hline & & CME-CV & $\begin{array}{l}\text { PSO } \\
\text { based } \\
\text { CME-CV }\end{array}$ & $\begin{array}{l}\text { ABC based } \\
\text { CME-CV }\end{array}$ & $\begin{array}{l}\text { WOA } \\
\text { based } \\
\text { CME-CV }\end{array}$ & $\begin{array}{l}\text { CME-C } \\
\text { V }\end{array}$ & $\begin{array}{l}\text { PSO } \\
\text { based } \\
\text { CME-CV }\end{array}$ & $\begin{array}{l}\text { ABC } \\
\text { based } \\
\text { CME-C } \\
\text { V } \\
\end{array}$ & $\begin{array}{l}\text { WOA } \\
\text { based } \\
\text { CME-C } \\
\text { V } \\
\end{array}$ \\
\hline $\begin{array}{l}\text { SIMULATED } \\
\text { INPUT-1 }\end{array}$ & 3.35 & 3.70 & 3.45 & 3.52 & 3.55 & 3.84 & 3.57 & 3.64 & 3.67 \\
\hline $\begin{array}{l}\text { SIMULATED } \\
\text { INPUT-2 }\end{array}$ & 7.32 & 7.60 & 7.55 & 7.57 & 7.54 & 8.22 & 8.16 & 8.19 & 8.24 \\
\hline $\begin{array}{l}\text { SIMULATED } \\
\text { INPUT-3 }\end{array}$ & 13.37 & 13.67 & 13.42 & 13.52 & 13.45 & 15.83 & 15.50 & 15.63 & 15.37 \\
\hline $\begin{array}{l}\text { SIMULATED } \\
\text { INPUT-4 }\end{array}$ & 17.7 & 18.00 & 17.87 & 17.92 & 17.86 & 21.95 & 21.75 & 21.83 & 21.77 \\
\hline $\begin{array}{l}\text { SIMULATED } \\
\text { INPUT-5 }\end{array}$ & 23.38 & 23.90 & 23.86 & 23.95 & 23.48 & 31.40 & 31.33 & 31.49 & 30.89 \\
\hline
\end{tabular}

Table-2: Comparison of optimal thresholding value

\begin{tabular}{|c|c|c|c|c|}
\hline INPUT & CME-CV & $\begin{array}{c}\text { PSO } \\
\text { based } \\
\text { CME-CV }\end{array}$ & $\begin{array}{c}\text { ABC } \\
\text { based } \\
\text { CME-CV }\end{array}$ & $\begin{array}{c}\text { WOA } \\
\text { based } \\
\text { CME-CV }\end{array}$ \\
\hline $\begin{array}{c}\text { SIMULATED } \\
\text { INPUT-1 }\end{array}$ & 90 & 82 & 84 & 89 \\
\hline $\begin{array}{c}\text { SIMULATED } \\
\text { INPUT-2 }\end{array}$ & 83 & 80 & 87 & 85 \\
\hline $\begin{array}{c}\text { SIMULATED } \\
\text { INPUT-3 }\end{array}$ & 77 & 75 & 76 & 79 \\
\hline $\begin{array}{c}\text { SIMULATED } \\
\text { INPUT-4 }\end{array}$ & 77 & 71 & 74 & 79 \\
\hline $\begin{array}{c}\text { SIMULATED } \\
\text { INPUT-5 }\end{array}$ & 76 & 78 & 78 & 80 \\
\hline
\end{tabular}

From the figure-4, delivers all the segmented output soil samples of PSO, ABC and SSA based CME-CV thresholding. It concise all the approaches to show the segmented in both the macropore and micropore. In table-3 visibly illuminates how the proposed methodology porosity value is closer to the ground truth porosity value compare than other approaches of with and with optimization techniques. WOA based CME-CV is shown its efficacy level of all the ranges of porous level. In fig 5 and 6 , is a comparison chart of relative porosity and misclassification error between the approaches such as CME-CV, PSO based CME-CV, ABC based CME-CV and WOA based CME-CV.

\section{CONCLUSION}

In this paper, an effective combination of Whale optimization Algorithm with Combined maximum entropy and class variance thresholding was proposed to enhance the accuracy of soil porosity examination. In this technique analysed with 50 simulated soil CT samples and with their ground truth images. The soil porous efficiency of simulated sample is tested with the well-known constraints such as soil pore or vacuum level, void-or air space ratio, relative porosity error and misclassification error. The proposed system was compared with bio-inspired approaches and without bio Inspired approaches. In terms of with bio-inspired approaches have attained improved porosity with respect to the ground truth image. In this comparative evaluation of PSO, ABC and WOA with CME-CV was conveyed their accuracy of porous level examination successfully. For the future that WOA based CME-CV has tested with some other modality of datasets and some other threshold based image segmentation.
It also investigate with the help of other efficient segmentation parameters. To improve the efficiency of the methodology will try with assured bio inspired optimization approaches.

\section{ACKNOWLEDGEMENTS}

The author would like to thank the Sir. C.V. RAMAN KRISHNAN International Research Centre for providing financial assistance under the University Research Fellowship. Also we thank the Department of Electronics and Communication Engineering of Kalasalingam Academy of Research and Education, Tamil Nadu, India for permitting to use the computational facilities available in Centre for Research in Signal Processing and VLSI Design which was set up with the support of the Department of Science and Technology (DST), New Delhi under FIST Program in 2013 (Reference No: SR/FST/ETI-336/2013 dated November 2013).

\section{REFERENCES}

1. Nobuyuki Otsu: A threshold selection method from gray-level Histogram. IEEE Transaction on Systems, Man, And Cybernetics, Vol. smc-9 (1979). 0018-9472/79/0100-0062\$00.75

2. Miller, B.A., Schaetzl, R.J.: History of Soil Geography in the Context of scale. Geoder-12090; (17) (2015)

3. Arunpandian. M., Arunprasath., T., Vishnuvarthanan., G., Pallikonda Rajasekaran. M.: Thresholding Based Soil Feature Extraction from Digital Image Samples - A Vision towards Smarter Agrology. Information and Communication Technology for Intelligent Systems Volume-1, Smart Innovation, Systems and Technologies 83, DOI 10.1007/978-3-319-63673-3_55 (2017)

4. Roy, A., \& Perfect, E. (2014). Lacunarity analyses of multifractal and natural grayscale patterns. Fractals, 22(03), 1440003.

5. Bhandari AK, Kumar A, Singh GK. Modified artificial bee colony based computationally efficient multilevel thresholding for satellite image segmentation using Kapur's, Otsu and Tsallis functions. Expert Systems with Applications. 2015 Feb 15; 42(3):1573-601.

6. Mafarja, M.M. and Mirjalili, S., 2017. Hybrid Whale Optimization Algorithm with simulated annealing for feature selection. Neurocomputing, 260, pp.302-312.

7. Mirjalili, S. and Lewis, A., 2016. The whale optimization algorithm. Advances in engineering software, 95, pp.51-67.

8. Faris H, Mafarja MM, Heidari AA, Aljarah I, Ala'M AZ, Mirjalili S, Fujita H. An efficient binary salp swarm algorithm with crossover scheme for feature selection problems. Knowledge-Based Systems. 2018 Aug 15; 154:43-67.

9. Hussien AG, Hassanien AE, Houssein EH. Swarming behavior of salps algorithm for predicting chemical compound activities. In2017 Eighth International Conference on Intelligent Computing and Information Systems (ICICIS) 2017 Dec 5 (pp. 315-320). IEEE.

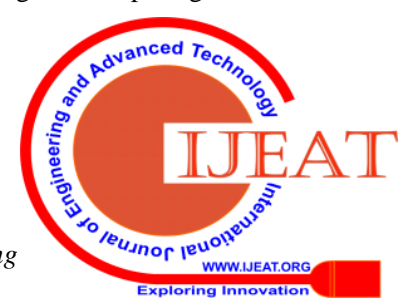


10. Wang H, Dong Y. An improved image segmentation algorithm based on otsu method. In International Symposium on Photo electronic Detection and Imaging 2007: Related Technologies and Applications 2008 Feb 19 (Vol. 6625, p. 66250I). International Society for Optics and Photonics.

11. Tseng CL, Alves MC, Crestana S. Quantifying physical and structural soil properties using X-ray micro tomography. Geoderma. 2018 May $15 ; 318: 78-87$.

12. Pires LF, Mooney SJ, Auler AC, Atkinson B, Sturrock CJ. X-ray micro tomography to evaluate the efficacy of paraffin wax coating for soil bulk density evaluation. Geoderma. 2019 Mar 1; 337:935-44.

13. Arunpandian M, Arunprasath T, Vishnuvarthanan G, Rajasekaran MP. Soil Porosity Analysis Using Combined Maximum Entropy and Class Variance Thresholding. InMicroelectronics, Electromagnetics and Telecommunications 2019 (pp. 641-650). Springer, Singapore.

14. Ma R, Cai C, Li Z, Wang J, Xiao T, Peng G, Yang W. Evaluation of soil aggregate microstructure and stability under wetting and drying cycles in two Ultisols using synchrotron-based X-ray micro-computed tomography. Soil and Tillage Research. 2015 Jun 1; 149:1-1.

\section{AUTHORS PROFILE}

Mr.M.Arunpandian, is a full time research scholar in the Department of Electronics and Communication Engineering at Kalasalingam Academy of Research and Education. He has completed his M.E in VLSI Design at Anna University in 2016, and B.E. in Electronics and Communication Engineering at Anna University in 2012, both located at Tamilnadu, India. His primary research focus is upon Real time image processing.

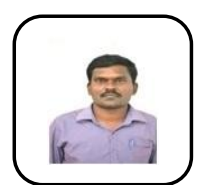

Dr.T.Arunprasath is an Associate professor in Department Biomedical Engineering at Kalasalingam Academy of Research and Education. he received his Ph.D. in Electronics and Communication Engineering from Kalasalingam University, Krishnankoil in 2015, his M.E. in Applied Electornics from Anna University in 2009 (Mohamed Sathak Engineering College) and his B.E. in Electrical and Electronics Engineering from Anna University (Syed Ammal Engineering College) in 2006. His research interests include biomedical instrumentation, image processing, image segmentation cloud computing, image segmentation. He has published 16 technical journals and 20 technical papers in refereed conferences in these areas. He is a life member of ISTE.

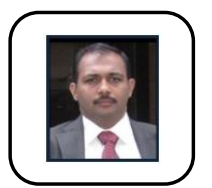

Dr. M. PallikondaRajasekaran, Born in Srivilliputhur, Virudhunagar District of Tamil Nadu in 1980, he had his schooling in the same town and graduated in Electronics and Instrumentation Engineering in 2001 from Shanmugha College of Engineering, Thanjavurand completed his M.Tech. Degree in 2002 with second Rank in SASTRA University. He pursued his doctoral programme in Anna University, Chennai. Starting as a Lecturer in 2003, he became Asst. Professor in 2008, Associate Professor in 2009 and Professor in 2012 in Kalasalingam Academy of Research and Education. He had a deep involvement in Bio-signal Processing research. His work on the Image Segmentation for identification of brain tumour and image reconstruction and compression using medical images for diagnosis. Over 150 B.Tech students, 75 M.Tech students, and 8 Doctorates stand testimony for his productivity in Image Processing, Wireless Sensor Networks, and Biomedical Instrumentation research. He has so far published more than 50 papers in national and international journals and conferences. He is a Fellow of Indian Society For Technical Education (ISTE), Institute of Electrical and Electronics Engineers (IEEE), Asia-Pacific Chemical, Biological\& Environmental Engineering Society (APCBEES), Institution of Engineers (India)(IE), International Association of Engineers (IAENG) and International Association of Computer Science and Information Technology (IACSIT).

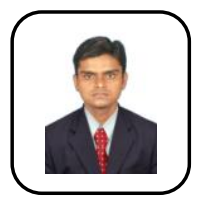

Dr.G.Vishnuvarthanan, born in 1986, has research stints in the avenues of medical image processing and artificial intelligence. He was awarded $\mathrm{PhD}$ in the year 2015 and bachelor's degree in Instrumentation and Control Engineering by 2007, and Master's Degree in VLSI by 2009. He has more than ten years of teaching and research experience and has his affiliation as Associate Professor with the Department of Biomedical Engineering of School of Bio and Chemical Sciences in the Kalasalingam Academy of Research and Education, Tamilnadu, India. 